\title{
A semi-analytical scheme for highly oscillatory integrals over tetrahedra
}

\author{
R. Hospital-Bravo, J. Sarrate and P. Díez * \\ Laboratori de Càlcul Numèric (LaCàN), ETS de Camins Canals i Ports de Barcelona, Universitat Politècnica de \\ Catalunya, Jordi Girona 1, E-08034 Barcelona, Spain
}

\begin{abstract}
SUMMARY
This paper details a semi-analytical procedure to efficiently integrate the product of a smooth function and a complex exponential over tetrahedral elements. These highly oscillatory integrals appear at the core of different numerical techniques. Here, the Partition of Unity Method (PUM) enriched with plane waves is used as motivation. The high computational cost or the lack of accuracy in computing these integrals is a bottleneck for their application to engineering problems of industrial interest. In this integration rule, the non-oscillatory function is expanded into a set of Lagrange polynomials. In addition, Lagrange polynomials are expressed as a linear combination of the appropriate set of monomials, whose product with the complex exponentials is analytically integrated, leading to 16 specific cases that are developed in detail. Finally, we present several numerical examples to assess the accuracy and the computational efficiency of the proposed method, compared to standard Gauss-Legendre quadratures.
\end{abstract}

KEY WORDS: highly oscillatory integral; semi-analytical integration rule; Lagrange polynomials; Helmholtz equation; Partition of Unity Method (PUM); plane waves

\section{INTRODUCTION}

During the last two decades special attention has been focused on the computation of highly oscillatory integrals in applied sciences and engineering. The problem arises in several fields such as quantum mechanics, image analysis, electrodynamics, and wave propagation problems. This type of integrals cannot be efficiently computed with standard quadratures (such as the Gauss-Legendre quadrature) because the highly oscillatory integrand is not properly represented by polynomial interpolation. Thus, a very large number of integrations points is needed and the computational cost becomes prohibitive, particularly in the case of medium or high frequencies, three-dimensional problems or large domains.

\footnotetext{
*Correspondence to: P. Díez, Laboratori de Càlcul Numèric (LaCàN), ETS de Camins Canals i Ports de Barcelona, Universitat Politècnica de Catalunya, Jordi Girona 1, E-08034 Barcelona, Spain.pedro.diez@upc.edu

Contract/grant sponsor: KIC InnoEnergy and European Institute of Innovation and Technology (EIT) through project Offshore Test Station (OTS) and Ministerio de Economía y Competitividad; contract/grant number: OTS; 03_2011_LH03 Industry Energy Efficiency, DPI2014-51844-C2-2-R, and CTM2014-55014-C3-3-R
} 
Wave problems lead to oscillatory solutions, which are poorly captured by the classical polynomial shape functions, associated with the standard Finite Element Method (FEM). Moreover, for high wavenumber applications, the numerical dispersion makes the discrete wavenumber differ drastically from the exact solution. This is called the pollution effect, and it separates the solution of the standard FEM from the best approximation [1] [2, Section 4.6] [3].

Although high-order methods and discontinuous formulations provide lower dissipation and dispersion, alternative finite element formulations have been developed to overcome this limitation by including special shape functions into the approximation space, see $[4,5]$ for continuous formulations or $[6,7,8,9]$ for discontinuous formulations. These enriching functions include a priori knowledge of the solution and improve the quality of the local and global approximation properties. In the case of the Helmholtz equation, it is advantageous to include sets of plane waves propagating in different directions, since they are free-space natural solutions when a uniform wavenumber is considered and they form a so-called c-complete set of functions that allows spanning the whole space of solutions, see [10, 11] for details. Thus, the enriched approximation space allows a drastic reduction of the pollution error, see [1] and [2, Section 4.8] for a detailed analysis. This alleviates the constraint of having a minimum number of elements per wavelength (typically 10 or 12 as a rule of thumb), allowing the use of coarser meshes with several wavelengths per element. These meshes provide a considerable reduction in the total number of unknowns (more than $90 \%$ in some cases).
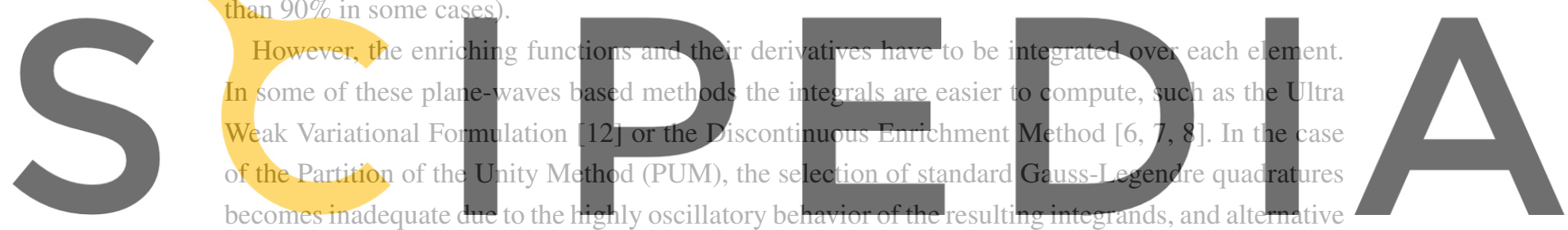

efficient integration techniques are needed. Many classical and modern methods for solving 1D finite

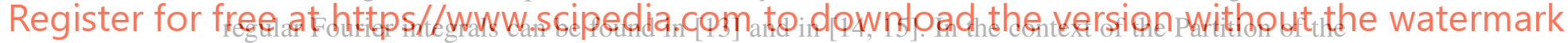

Unity Method (PUM), Ortiz and Sanchez [16] introduced a local coordinate rotation over triangular elements to obtain ID osciliatory integrands and compute these integrais semi-analyticaily. Bettess et al. $[17,18]$ developed semi-analytical rules for 2D problems that consider the special nature of the integrand and profit from it to perform the integration. In these rules, the non-oscillatory part of the integrands is approximated by a set of interpolating Lagrange polynomials. Then, the products of these polynomials and the complex exponentials are integrated analytically. The result is a set of integration weights, which are specific for each combination of element geometry and parameter of the complex exponential. The key point of these rules is that the distribution of the integration points captures the behavior of the smooth part of the integrand, rather than the whole oscillatory behavior, which is analytically captured by the integration weights. Therefore, the spacing of the integration points is larger, dramatically reducing their number and, hence, the number of function evaluations, compared to the Gauss-Legendre quadrature. This approach has been recently applied in the context of underwater acoustics in [19]. Gabard [20] presented an alternative approach to compute the integral of the product of a polynomial and a complex exponential over arbitrary polygons, polyhedral volumes and 3D surfaces, by rewriting volume integrals in terms of 1D integrals along the element edges thanks to the Gauss and the Stokes theorems. 
The novelty of our contribution is a fast and robust semi-analytical rule to compute the volumetric integrals of highly oscillatory functions (expressed as the product of a smooth function and a complex exponential) on tetrahedral elements. In this sense, it is a generalization to a three dimensional setting of the integration rule developed by [17, 18]. The method proposed here follows the usual practice of standard quadratures and considers the evaluation of the non-oscillatory function at the integration points. This is in contrast to [20] that considers as input data the coefficients of the polynomial (non-oscilatory part of the integrand), and after applying the Gauss and the Stokes theorems, performs one dimensional integrals along the element edges. The proposed quadrature is better fitted to be implemented in a Finite Element like methodology. This is because the information describing the (non-oscillatory part of the) function to be integrated is required as values at a set of integration points, which is the standard practice in Finite Elements. On the contrary, the quadrature introduced in [20] requires as input data the coefficients of the polynomial in some basis. Thus, for the specific application in a Finite Element framework, using [20] requires an additional step that consists in finding the analytical expression of the polynomial from point values with some interpolation technique. Note that this new operation introduces an additional interpolation error.

The rest of the paper is organized as follows: Section 2 describes the semi-analytical scheme, detailing the Lagrange expansion in terms of a linear combination of a basis of monomials, and the expression of the integration weights of the semi-analytical rule. Section 3 provides several preliminary results required to develop Section 4, which presents the new schene to compute
the target integral. Specifically, we detail how this scheme leads to 16 different cases. Section 5
summarizes how the PUM enriched with plane waves bencfits from this semi-analytical scheme.
Section 6 presents several numerical examples to underline the main properties of the proposed 4 Register for free at https//www.scipedia.com to download the version without the watermark

\section{HIGHLY OSCILLATORY INTEGRAL OVER A TETRAHEDRON}

The objective of this work is obtaining an efficient semi-analytical integration rule to compute the following family of highly oscillatory integrals over a tetrahedron $\Omega^{\mathrm{TET}}$ :

$$
K=\int_{\Omega^{\mathrm{TET}}} f(\boldsymbol{x}) \exp (i \boldsymbol{v} \cdot \boldsymbol{x}) \mathrm{d} \boldsymbol{x},
$$

where $\boldsymbol{x}$ is the vector of coordinates, $f$ is a smooth (non-oscillatory) function, $i=\sqrt{-1}$ is the imaginary unit, and $\boldsymbol{v}$ is an arbitrary and complex vector. The complex exponential is considered as highly oscillatory if it oscillates several times inside the element, that is, if

$$
\frac{\|\boldsymbol{v}\| h}{2 \pi} \gg 1
$$

where $h$ is a characteristic length. Since the methodology is conceived for an application in the context of generalized finite elements, in the following $h$ is denoted as the element size. This kind of integral is also known as regular finite Fourier integral. 


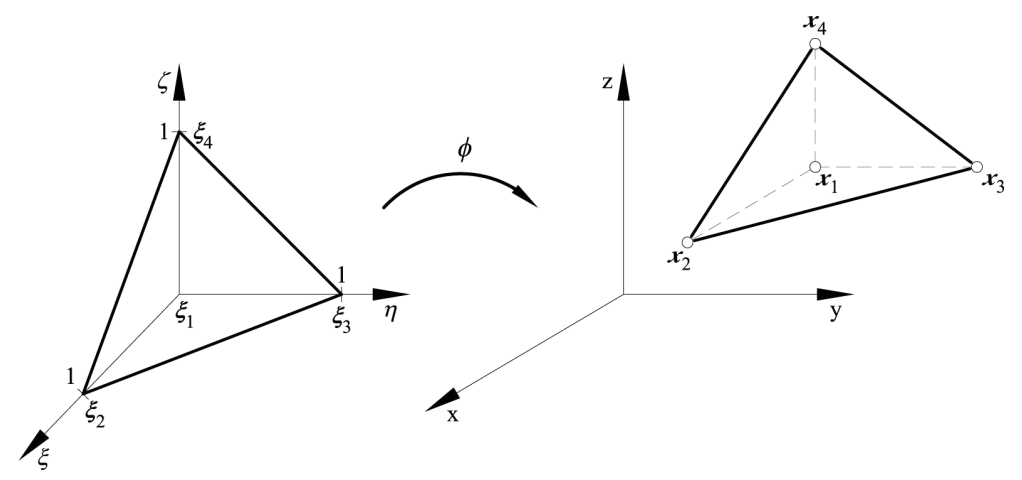

Figure 1. Geometrical mapping between the reference and physical tetrahedral element.

\subsection{Lagrange polynomials expansion and semi-analytical scheme}

We rewrite the integral in Equation (1) at the reference tetrahedron $\Omega^{\text {ref }}$, see Figure 1, as

$$
K=\int_{\Omega^{\text {ref }}} f(x(\xi)) \exp (i v \cdot x(\xi))|J(\xi)| \mathrm{d} \xi=\int_{\Omega^{\text {ref }}} F(\xi) \exp (i v \cdot x(\xi)) \mathrm{d} \xi,
$$
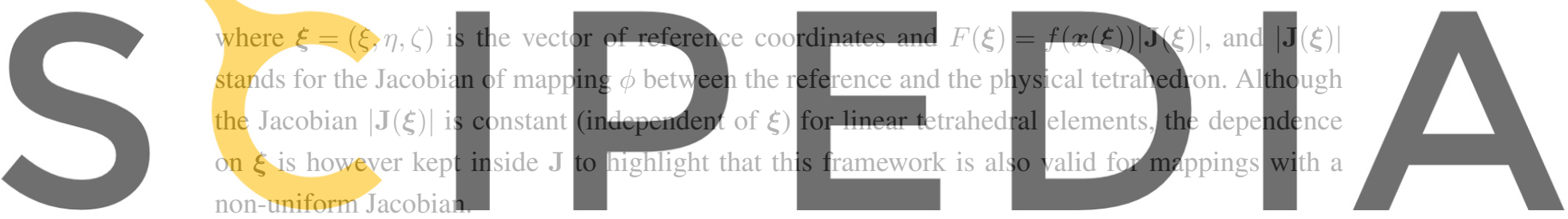

The non-oscillatory function $F(\xi)$ is now approximated by means of a set of Lagrange Register for freepaitinttposydywiw.scipedia.com to download the version without the watermark

$$
F(\xi) \approx \sum_{d=1}^{n_{p}} F\left(\xi_{d}\right) L_{d}(\xi)
$$

where $L_{d}$ is the Lagrange polynomial of degree $p$ associated with the $d$-th integration point $\boldsymbol{\xi}_{d}$, $d=1, \ldots, n_{p}$, and $n_{p}$ is the number of integration points. Therefore, the integral in Equation (1) is computed using the following integration rule:

$$
K \approx \int_{\Omega^{\text {ref }}}\left(\sum_{d=1}^{n_{p}} F\left(\boldsymbol{\xi}_{d}\right) L_{d}(\boldsymbol{\xi})\right) \exp (i \boldsymbol{v} \cdot \boldsymbol{x}(\boldsymbol{\xi})) \mathrm{d} \boldsymbol{\xi}=\sum_{d=1}^{n_{p}} F\left(\boldsymbol{\xi}_{d}\right) w_{d},
$$

where

$$
w_{d}=\int_{\Omega^{\mathrm{ref}}} L_{d}(\boldsymbol{\xi}) \exp (i \boldsymbol{v} \cdot \boldsymbol{x}(\boldsymbol{\xi})) \mathrm{d} \boldsymbol{\xi} \quad \text { for } d=1, \ldots, n_{p},
$$

is the complex integration weight corresponding to the $d$-th integration point. Note that the accuracy of integral (1) strongly depends on the quality of the approximation in Equation (3).

The difficulty lies now in the evaluation of the integration weights $w_{d}$, for $d=1, \ldots, n_{p}$. Their values depend on vector $\boldsymbol{v}$ and the element geometry. Therefore, it is not possible to a priori compute a set of weights for a given set of integration points that are valid for any element geometry. To develop an efficient procedure, it is convenient to decompose the Lagrange polynomials into the 
appropriate set of 3D monomials:

$$
L_{d}(\boldsymbol{\xi})=\sum_{|\boldsymbol{a}| \leq p} \lambda_{d, \boldsymbol{a}} M_{\boldsymbol{a}}(\boldsymbol{\xi})
$$

where

$$
M_{\boldsymbol{a}}(\boldsymbol{\xi})=\xi^{a} \eta^{b} \zeta^{c}
$$

and $\lambda_{d, \boldsymbol{a}}$ is the coefficient associated with the $d$-th polynomial and the $\boldsymbol{a}$-th monomial. The multiindex notation considers $\boldsymbol{a}=(a, b, c)$, where $a, b, c$ are non-negative integers and $|\boldsymbol{a}|:=a+b+c \leq$ $p$. Appendix A details a procedure to compute the coefficients $\lambda_{d, a}$ of the monomial decomposition in a straightforward manner.

Substituting Equation (5) into Equation (4), we obtain a new expression for the integration weights:

$$
w_{d}=\sum_{|a| \leq p} \lambda_{d, a} \int_{\Omega^{\mathrm{ref}}} M_{a}(\boldsymbol{\xi}) \exp (i \boldsymbol{v} \cdot \boldsymbol{x}(\boldsymbol{\xi})) \mathrm{d} \boldsymbol{\xi} .
$$

\section{Remark 1}

Other interpolation functions could be used to approximate $F(\boldsymbol{\xi})$ in Equation (3). Nevertheless, Lagrange polynomials are easily decomposed into a set of monomials, see Appendix A, leading to the integration of products of monomials and complex exponential that can be performed semi-
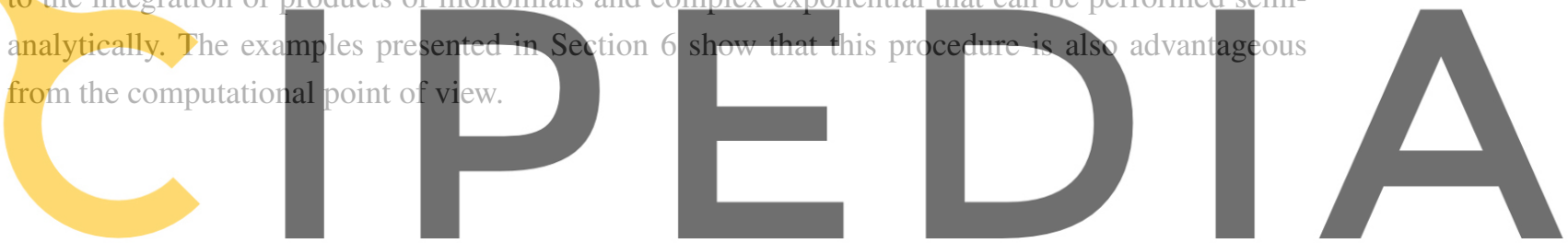

Remark 2

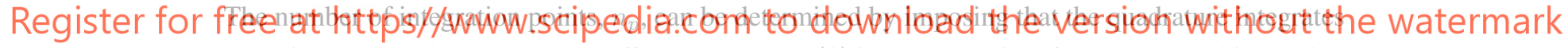

Equation (2) exactly (up to round-off errors) when $F(\xi)$ is a polynomial of degree $\leq p$. This number

must coincide with the dimension of the polynomial space, which can be obtained from the Pascal's

pyramid as

$$
n_{p}=\frac{1}{2}\left(\frac{(p+1)^{3}}{3}+(p+1)^{2}+\frac{4(p+1)}{6}\right) .
$$

If $F(\boldsymbol{\xi})$ is not a polynomial function, an appropriate value for $p$ fitting the behavior of the function has to be selected.

\section{Remark 3}

Following the work of Bettess et al. [17, 18], we consider an equidistributed set of integration points. Selecting optimal locations for the integration points as in the case of the Gauss-Legendre quadratures is not possible in this context. This is because the oscillating term, $\exp (i \boldsymbol{v} \cdot \boldsymbol{x}(\boldsymbol{\xi}))$, acting as kernel of a bilinear form that should be a scalar product, is a complex function. Consequently, it cannot be used to define a scalar product (a complex kernel function is associated with non-positive definite bilinear form). Even if the kernel was real valued, it would be dependent on the geometry of the element and on the argument of the complex exponential, vector $v$. 


\subsection{Tetrahedral finite elements}

We consider the 3D linear mapping $\phi$ from the reference coordinates $\boldsymbol{\xi}$ to the global coordinates $\boldsymbol{x}$ :

$$
\boldsymbol{x}=\boldsymbol{\phi}(\boldsymbol{\xi})=\boldsymbol{\alpha} \xi+\boldsymbol{\beta} \eta+\gamma \zeta+\boldsymbol{\delta}
$$

where

$$
\boldsymbol{\alpha}=\boldsymbol{x}_{2}-\boldsymbol{x}_{1}, \quad \boldsymbol{\beta}=\boldsymbol{x}_{3}-\boldsymbol{x}_{1}, \quad \boldsymbol{\gamma}=\boldsymbol{x}_{4}-\boldsymbol{x}_{1}, \quad \boldsymbol{\delta}=\boldsymbol{x}_{1},
$$

defined on the reference domain $0 \leq \xi \leq 1-\zeta-\eta, 0 \leq \eta \leq 1-\zeta$, and $0 \leq \zeta \leq 1$, see Figure 1 . Introducing equations (6) and (8) into the expression for the integration weights, Equation (7), we obtain

$$
w_{d}=\exp (i D) \sum_{|a| \leq p} \lambda_{d, \boldsymbol{a}} I_{\boldsymbol{a}}
$$

\section{where}

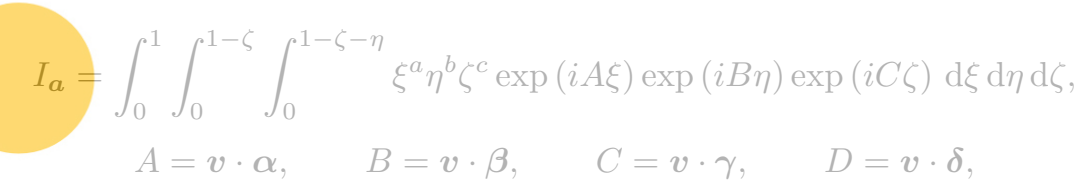

$$
A=v \cdot \alpha, \quad B=v \cdot \beta, \quad C=v \cdot \gamma, \quad D=v \cdot \delta,
$$

and $a, b, c$ being non-negative integers. Note that the weights depend on vector $v$ and the element
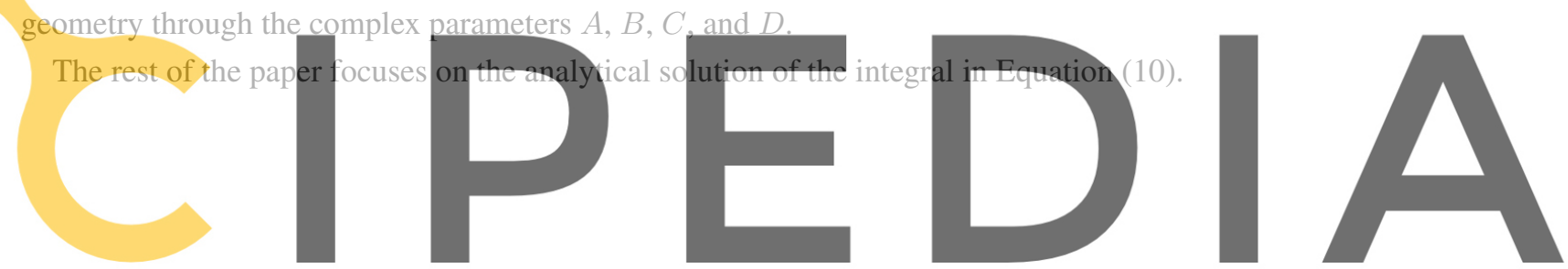

This section provides two basic results that will be used in Section 4 to obtain the analytical solution of integral (10). Our semi-analytical procedure seeks successive 1D term collections that head toward the analytical integration of the following one-dimensional product of a monomial and a highly oscillatory term:

$$
I_{1 \mathrm{D}}=\int s^{\nu} \exp (i \mu s) \mathrm{d} s
$$

where $\nu$ is a non-negative integer, and the value of the complex coefficient $\mu$ depends on the values of coefficients $A, B$ and $C$.

We consider this type of one-dimensional integral at two stages of the development of our numerical procedure. On the one hand, the analytical manipulation of Equation (10) leads to 1D highly oscillatory integrals such that the upper limit of the integral depends on the reference spatial coordinates, see details in Section 4. On the other hand, these intermediate integrals are further developed leading to 1D highly oscillatory integrals with fixed integration limits (between 0 and 1). This section focuses on the first situation while Appendix B deals with the second one.

We consider two cases in the first situation, depending on the value of parameter $\mu$, as suggested in [18]. First, if the modulus of $\mu$ is not small, $\mu \nrightarrow 0$, and given that exponent $\nu$ is a non-negative 
integer, after recursively applying the integration by parts theorem we have

$$
\int s^{\nu} \exp (i \mu s) \mathrm{d} s=\nu ! \sum_{r=0}^{\nu} \frac{i^{r-1} s^{\nu-r} \exp (i \mu s)}{(\nu-r) ! \mu^{r+1}} .
$$

Second, for certain combinations of the values of coefficients $A, B$ and $C$ in Equation (10), the analytical manipulations involve integrals of type (11) with a very small values of coefficient $\mu$, $\mu \rightarrow 0$ and Equation (12) provides inaccurate results. Thus, it is preferable to apply a truncated Taylor's expansion to the exponential function before proceeding with the analytical integration. Specifically, we set

$$
\exp (i \mu s) \approx \sum_{r=0}^{r_{\max }} \frac{(i \mu s)^{r}}{r !}
$$

where $r_{\max }$ is the number of terms retained in the approximation. This number is adjusted so that the truncation error in the series is of the order of the machine accuracy $\epsilon$. Since in this paper both $\nu$ and $r$ are non-negative integer parameters, we have

$$
\int s^{\nu} \exp (i \mu s) \mathrm{d} s \approx \sum_{r=0}^{r_{\max }} \frac{(i \mu)^{r}}{r !} \int s^{\nu+r} \mathrm{~d} s=\sum_{r=0}^{r_{\max }} \frac{(i \mu)^{r}}{r !} \frac{s^{\nu+r+1}}{\nu+r+1} .
$$
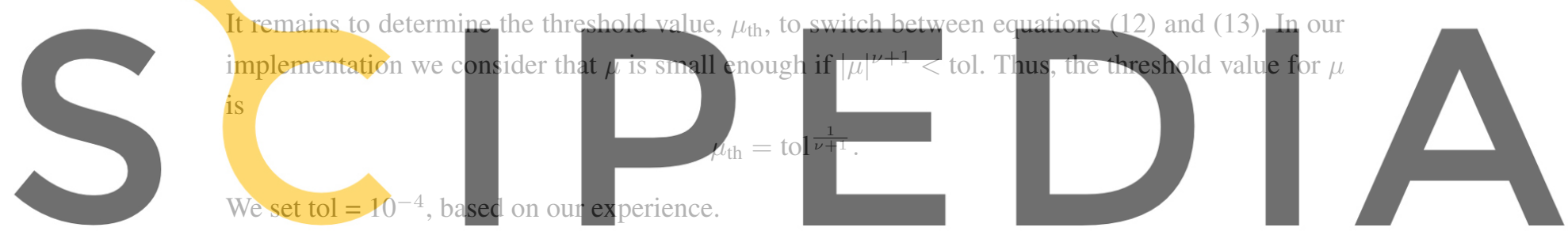

Finally, the following type of integral also appears in Section 4:

\section{Register for free at https//www.scipedia.com to download the version without the watermark $\int s^{\nu}(t-s)^{k} \exp (i \mu s) \mathrm{d} s$.}

This integral can be expressed as a sum of integrals from Equation (11) thanks to the binomial theorem,

$$
(t-s)^{\kappa}=\kappa ! \sum_{r=0}^{\kappa} \frac{(-1)^{r}}{(\kappa-r) ! r !} t^{(\kappa-r)} s^{r}
$$

leading to

$$
\int s^{\nu}(t-s)^{\kappa} \exp (i \mu s) \mathrm{d} s=\kappa ! \sum_{r=0}^{\kappa} \frac{(-1)^{r} t^{\kappa-r}}{(\kappa-r) ! r !} \int s^{\nu+r} \exp (i \mu s) \mathrm{d} s
$$

that can be solved using equations (12) or (13), depending on the value of coefficient $\mu$.

\section{ANALYTICAL DEVELOPMENT TO OBTAIN THE EXPRESSIONS FOR THE WEIGHTS}

To obtain the integration weights (9) of the semi-analytical rule, in this Section we develop a procedure to evaluate the highly oscillatory integral in Equation (10). Specifically, we first apply 
Table I. Possible combinations of values for coefficients $A, B$, and $C$ to obtain integral $I_{\mathbf{a}}$ from Equation (10) leading to 16 cases of analytical expressions

\begin{tabular}{c|cccccc}
\hline Case & $A$ & $B$ & $C$ & $C-B$ & $C-A$ & $B-A$ \\
\hline 1 & $\nrightarrow 0$ & $\nrightarrow 0$ & $\nrightarrow 0$ & $\nrightarrow 0$ & $\nrightarrow 0$ & $\nrightarrow 0$ \\
2 & $\nrightarrow 0$ & $\nrightarrow 0$ & $\rightarrow 0$ & - & - & $\nrightarrow 0$ \\
3 & $\nrightarrow 0$ & $\nrightarrow 0$ & $\nrightarrow 0$ & $\nrightarrow 0$ & $\rightarrow 0$ & $\nrightarrow 0$ \\
4 & $\nrightarrow 0$ & $\nrightarrow 0$ & $\nrightarrow 0$ & $\rightarrow 0$ & $\nrightarrow 0$ & $\nrightarrow 0$ \\
5 & $\nrightarrow 0$ & $\nrightarrow 0$ & $\nrightarrow 0$ & $\rightarrow 0$ & $\rightarrow 0$ & $\nrightarrow 0$ \\
6 & $\nrightarrow 0$ & $\rightarrow 0$ & $\nrightarrow 0$ & - & $\nrightarrow 0$ & - \\
7 & $\nrightarrow 0$ & $\rightarrow 0$ & $\nrightarrow 0$ & - & $\rightarrow 0$ & - \\
8 & $\nrightarrow 0$ & $\rightarrow 0$ & $\rightarrow 0$ & - & - & - \\
9 & $\nrightarrow 0$ & $\nrightarrow 0$ & $\nrightarrow 0$ & - & $\nrightarrow 0$ & $\rightarrow 0$ \\
10 & $\nrightarrow 0$ & $\nrightarrow 0$ & $\nrightarrow 0$ & - & $\rightarrow 0$ & $\rightarrow 0$ \\
11 & $\nrightarrow 0$ & $\nrightarrow 0$ & $\rightarrow 0$ & - & - & $\rightarrow 0$ \\
12 & $\rightarrow 0$ & $\nrightarrow 0$ & $\nrightarrow 0$ & $\nrightarrow 0$ & - & - \\
13 & $\rightarrow 0$ & $\nrightarrow 0$ & $\nrightarrow 0$ & $\rightarrow 0$ & - & - \\
14 & $\rightarrow 0$ & $\rightarrow 0$ & $\rightarrow 0$ & - & - & - \\
15 & $\rightarrow 0$ & $\rightarrow 0$ & $\nrightarrow 0$ & - & - & - \\
16 & $\rightarrow 0$ & $\rightarrow 0$ & $\rightarrow 0$ & - & - & -
\end{tabular}

the analytical expressions presented in the previous section, Equations (12) and (13), to perform the inner integrals involving $\xi$ and $\eta$. This procedure leads to 16 cases depending on the values of coefficients $A, B$ and $C$, and their respective differences, see Table I. In all of them, we finally
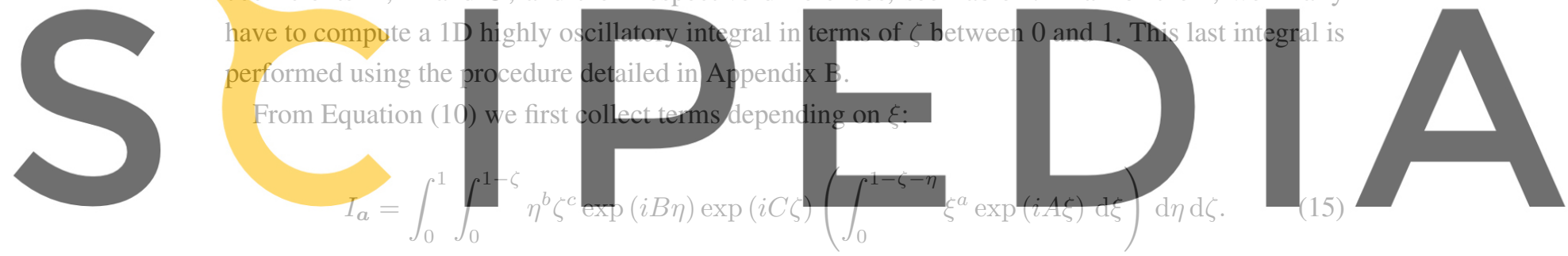

Register for free at https / /www scipedia. com to download the version without the watermark

in Section 3. Thus, its evaluation depends on the value of $A$. Cases 1-11 in Table I consider $A \nrightarrow 0$

while cases $12-16$ consider $A \rightarrow 0$.

4.1. Cases from 1 to $11(A \nrightarrow 0)$

If $A \nrightarrow 0$, we perform the 1D integral with respect to $\xi$ by means of Equation (12),

$$
\begin{aligned}
I_{\boldsymbol{a}}= & \int_{0}^{1} \int_{0}^{1-\zeta} \eta^{b} \zeta^{c} \exp (i B \eta) \exp (i C \zeta)\left[a ! \exp (i A \xi) \sum_{r=0}^{a} \frac{i^{r-1} \xi^{a-r}}{(a-r) ! A^{r+1}}\right]_{0}^{1-\zeta-\eta} \mathrm{d} \eta \mathrm{d} \zeta \\
= & a ! \int_{0}^{1} \int_{0}^{1-\zeta} \eta^{b} \zeta^{c} \exp (i B \eta) \exp (i C \zeta) \\
& \left(\exp (i A(1-\zeta-\eta)) \sum_{r=0}^{a}\left(\frac{i^{r-1}(1-\zeta-\eta)^{a-r}}{(a-r) ! A^{r+1}}\right)-\lim _{\xi \rightarrow 0}\left(\exp (i A \xi) \sum_{r=0}^{a} \frac{i^{r-1} \xi^{a-r}}{(a-r) ! A^{r+1}}\right)\right) \mathrm{d} \eta \mathrm{d} \zeta \\
= & a !\left(\exp (i A) \sum_{r=0}^{a}\left(\frac{i^{r-1}}{(a-r) ! A^{r+1}} I_{1, r}\right)-\frac{i^{a-1}}{A^{a+1}} I_{T R I}\right)
\end{aligned}
$$


where

$$
I_{1, r}=\int_{0}^{1} \int_{0}^{1-\zeta} \eta^{b} \zeta^{c}(1-\zeta-\eta)^{a-r} \exp (i(B-A) \eta) \exp (i(C-A) \zeta) \mathrm{d} \eta \mathrm{d} \zeta
$$

and

$$
I_{\mathrm{TRI}}=\int_{0}^{1} \int_{0}^{1-\zeta} \eta^{b} \zeta^{c} \exp (i B \eta) \exp (i C \zeta) \mathrm{d} \eta \mathrm{d} \zeta
$$

Equation (18) is the 2D version over triangular elements of the integral in Equation (10). The semi-analytical rule for this integral was proposed in [17], and it is detailed in Appendix C for completeness.

Taking into account Equation (14), and collecting the terms of $\eta$, Equation (17) becomes

$$
\begin{aligned}
I_{1, r}= & (a-r) ! \sum_{s=0}^{a-r}\left(\frac{(-1)^{s}}{(a-r-s) ! s !}\right. \\
& \left.\int_{0}^{1} \zeta^{c}(1-\zeta)^{a-r-s} \exp (i(C-A) \zeta)\left(\int_{0}^{1-\zeta} \eta^{b+s} \exp (i(B-A) \eta) \mathrm{d} \eta\right) \mathrm{d} \zeta\right) .
\end{aligned}
$$

At this point, another split in the procedure is produced depending on the value of $B-A$.
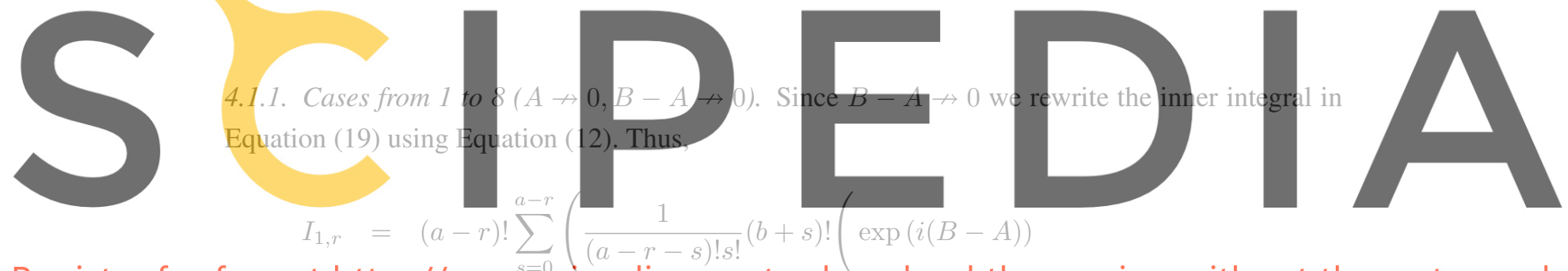

Register for free at https///wwww.scipedia.com to download the version without the watermark

$$
\begin{aligned}
& \sum_{t=0}\left(\frac{i^{2 s+t-1}}{(b+s-t) !(B-A)^{t+1}} \int_{0}^{1} \zeta^{c}(1-\zeta)^{a+b-r-t} \exp (i(C-B) \zeta) \mathrm{d} \zeta\right) \\
& \left.\left.-\frac{i^{b+s-1}}{(B-A)^{b+s+1}} \int_{0}^{1} \zeta^{c}(1-\zeta)^{a-r-s} \exp (i(C-A) \zeta) \mathrm{d} \zeta\right)\right) .
\end{aligned}
$$

Eight different cases arise depending on the possible combinations of $B, C, C-A$ and $C-B$. The expressions for these integrals are obtained by developing equations (18) and (20), using the results presented in Appendixes C and B, respectively.

4.1.2. Cases from 9 to $11(A \nrightarrow 0, B-A \rightarrow 0)$. Since $B-A \rightarrow 0$, we develop the inner integral in Equation (19) using Equation (13). Thus,

$$
\begin{aligned}
I_{1, r}= & (a-r) ! \sum_{s=0}^{a-r}\left(\frac{1}{(a-r-s) ! s !}\right. \\
& \left.\sum_{t=0}^{t_{\max }}\left(\frac{i^{2 s+t}(B-A)^{t}}{t !(b+s+t+1)} \int_{0}^{1} \zeta^{c}(1-\zeta)^{a+b-r+t+1} \exp (i(C-A) \zeta) \mathrm{d} \zeta\right)\right) .
\end{aligned}
$$


Three different cases arise depending on the possible combinations of $C$ and $C-A$. Again, integral (18) is computed using the procedure detailed in Appendix C. The expressions for Equation (21) can be obtained by using the binomial theorem, Equation (14), and Appendix B.

\subsection{Cases from 12 to $16(A \rightarrow 0)$}

Starting again from Equation (15), and considering that $A \rightarrow 0$, we perform the 1D integral with respect to $\xi$ using Equation (13). Hence,

$$
I_{\boldsymbol{a}}=\sum_{r=0}^{r_{\max }} \frac{(i A)^{r}}{r !(a+r+1)} \int_{0}^{1} \int_{0}^{1-\zeta} \eta^{b} \zeta^{c}(1-\zeta-\eta)^{a+r+1} \exp (i B \eta) \exp (i C \zeta) \mathrm{d} \eta \mathrm{d} \zeta .
$$

Applying the binomial theorem, Equation (14), and collecting terms depending on $\eta$, we get

$$
I_{a}=\sum_{r=0}^{r_{\max }}\left(\frac{A^{r}(a+r) !}{r !} \sum_{s=0}^{a+r+1} \frac{i^{r+2 s}}{(a+r-s+1) ! s !} I_{2, r s}\right) \text {, }
$$

where
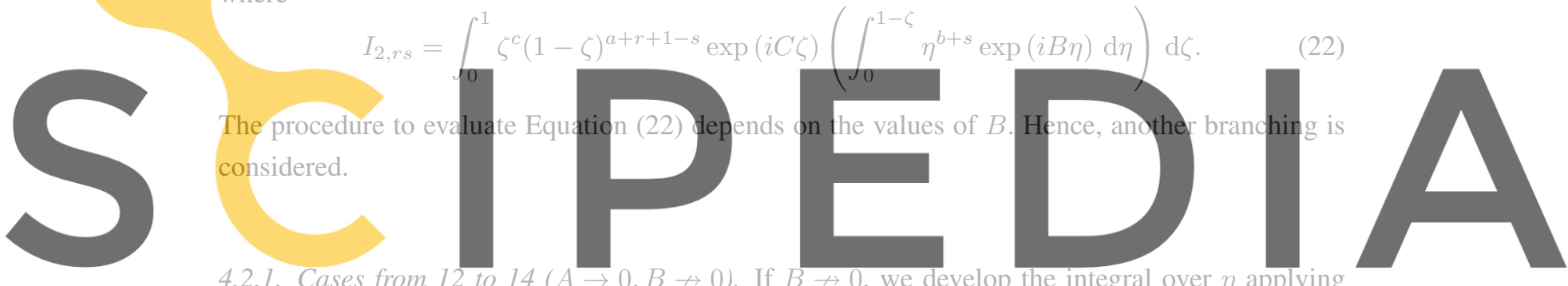

free at httips//www.scipedia.com to download the version without the watermark

$$
\begin{aligned}
I_{2, r s}= & (b+s) !\left(\exp (i B) \sum_{t=0}^{b+s} \frac{i^{t-1}}{(b+s-t) ! B^{i+1}} \int_{0}^{1} \zeta^{c}(1-\zeta)^{a+b+r-t+1} \exp (i(C-B \zeta) \mathrm{d} \zeta\right. \\
& \left.-\frac{i^{b+s-1}}{B^{b+s+1}} \int_{0}^{1} \zeta^{c}(1-\zeta)^{a+r-s+1} \exp (i C \zeta) \mathrm{d} \zeta\right) .
\end{aligned}
$$

Three cases have to be considered depending on the values of $C$ and $C-B$. All of them are solved by first using the binomial theorem, Equation (14), second applying Equation (12) and/or Equation (13), and finally using Appendix B.

4.2.2. Cases 15 and $16(A \rightarrow 0, B \rightarrow 0)$. If $B \rightarrow 0$, the integral over $\eta$ in Equation (22) is carried out using Equation (13). Thus,

$$
I_{2, r s}=\sum_{t=0}^{t_{\max }} \frac{(i B)^{t}}{t !(b+s+t+1)} \int_{0}^{1} \zeta^{c}(1-\zeta)^{a+b+r+t+2} \exp (i C \zeta) \mathrm{d} \zeta .
$$

After applying the binomial theorem, Equation (14), both cases 15 and 16 are solved by using the corresponding method in Appendix B. 


\section{EXAMPLE OF A PRACTICAL APPLICATION: THE PUM ENRICHED WITH PLANE WAVES}

An important application of the semi-analytical rule presented in this work is the computation of the highly oscillatory integrals that appear when the Helmholtz equation is solved using the Partition of Unity Method enriched with plane waves. The Helmholtz equation is the time-harmonic version of the wave equation, namely:

$$
\Delta u+k^{2} u=0 \text { in } \Omega
$$

where $k$ is the wavenumber, $\Delta$ is the Laplace operator, $\Omega$ is the problem domain, and the unknown $u$ is, for instance, the acoustic pressure, the wave height, or the electro-magnetic potential. Equation (25) is complemented by a Robin boundary condition

$$
\frac{\partial u}{\partial n}-\tau k u=g \quad \text { in } \Gamma=\partial \Omega,
$$

where $n$ is the outward normal to the boundary, $\tau$ is the complex transmission coefficient and $g$ is the Robin independent term. We assume that domain $\Omega$ is discretized into a tetrahedral mesh composed of $n_{n}$ nodes. The weak form of the problem is stated as: find $u_{h, q} \in \mathcal{W}_{h, q}$ such that

$$
\int_{\Omega}\left(k^{2} u_{h, q} \bar{v}-\nabla u_{h, q} \cdot \nabla \bar{v}\right) \mathrm{d} \Omega+\int_{\Gamma} \tau k u_{h, q} \bar{v} \mathrm{~d} \Gamma=-\int_{\Gamma} g \bar{v} \mathrm{~d} \Gamma, \quad \forall v \in \mathcal{W}_{h, q},
$$

where ${ }^{\top}$ denotes complex conjugate,

$$
\mathcal{W}_{h, q}=\left\{v \mid v=\sum_{m=1}^{n_{n}} N_{m}\left(\sum_{r=1}^{q} a_{m, r} W_{m, r}\right)\right\}
$$

is the solution space corresponding to a mesh characterized by an element size $h$ and $q$ plane waves (enrichment functions) pasted at each node (patch), $N_{m}$ is the standard first-order polynomial shape function (hat function) associated with the $m$-th node, and $a_{m, r}$ is the unknown complex coefficient associated with the $r$-th plane wave at the $m$-th node, namely

$$
W_{m, r}(\boldsymbol{x})=\exp \left(i k_{m} \boldsymbol{e}_{r} \cdot\left(\boldsymbol{x}-\boldsymbol{x}_{m}\right)\right)
$$

$k_{m}$ and $\boldsymbol{x}_{m}$ being the wavenumber and coordinates vector of the $m$-th node, and $\boldsymbol{e}_{r}$ the unit vector corresponding to the $r$-th plane wave direction.

According to equations (28) and (29), the PUM approximation has the form

$$
u_{h, q}(\boldsymbol{x})=\sum_{m=1}^{n_{n}} \sum_{r=1}^{q} a_{m, r} \phi_{m, r}(\boldsymbol{x})
$$

where $\phi_{m, r}$ is the approximation function associated with the $m$-th node and the $r$-th direction,

$$
\phi_{m, r}(\boldsymbol{x})=N_{m}(\boldsymbol{x}) W_{m, r}(\boldsymbol{x})=N_{m}(\boldsymbol{x}) \exp \left(i k_{m} \boldsymbol{e}_{r} \cdot\left(\boldsymbol{x}-\boldsymbol{x}_{m}\right)\right)
$$


The surface integrals appearing in Equation (27) can be computed using the 2D methods proposed in $[17,18]$. Here we focus on the efficient and accurate evaluation of the volume integral. Specifically, the volumetric elemental contribution to the system matrix is obtained by introducing this set of approximation functions into the weak form of the problem. Thus, integrating over the element $\Omega^{(e)}$ we get

$$
\begin{aligned}
K_{m r, n s}^{(e)} & =\int_{\Omega^{(e)}}\left[k^{2} \phi_{n, s} \bar{\psi}_{m, r}-\nabla \phi_{n, s} \cdot \boldsymbol{\nabla} \bar{\psi}_{m, r}\right] \mathrm{d} \boldsymbol{x} \\
& =\exp \left(i\left(\bar{k}_{m} \boldsymbol{e}_{r} \cdot \boldsymbol{x}_{m}-k_{n} \boldsymbol{e}_{s} \cdot \boldsymbol{x}_{n}\right)\right) \int_{\Omega^{(e)}} f_{m r, n s}(\boldsymbol{x}) \exp \left(i\left(-\bar{k}_{m} \boldsymbol{e}_{r}+k_{n} \boldsymbol{e}_{s}\right) \cdot \boldsymbol{x}\right) \mathrm{d} \boldsymbol{x}
\end{aligned}
$$

where $m, n=1, \ldots, n_{n}, r, s=1, \ldots, q$,

$$
f_{m r, n s}(\boldsymbol{x})=k^{2}(\boldsymbol{x}) N_{m}(\boldsymbol{x}) N_{n}(\boldsymbol{x})-\left(\boldsymbol{\nabla} N_{m}(\boldsymbol{x})-i \bar{k}_{m} N_{m}(\boldsymbol{x}) \boldsymbol{e}_{r}\right) \cdot\left(\boldsymbol{\nabla} N_{n}(\boldsymbol{x})+i k_{n} N_{n}(\boldsymbol{x}) \boldsymbol{e}_{s}\right)
$$

is a non-oscillatory function, and $\psi_{m, r}$ is the test function associated with the $m$-th node and the $r$-th direction, which has the same form as the approximation function $\phi_{m, r}$ in Equation (30). Note that the integral in Equation (31) is a particular case of Equation (1) with $\boldsymbol{v}=-\bar{k}_{m} \boldsymbol{e}_{r}+k_{n} \boldsymbol{e}_{s}$.

\section{NUMERICAL EXPERIMENTS}

In this section we present three examples that involve the integration of highly oscillatory functions over tetrahedra, in order to test the semi-analytical integration rule proposed in this work. Specifically, we compare its performance with the one of the standard Gauss-Legendre quadrature.

In the first example, several non-oscillatory functions are considered and the product of each of them and a complex exponential is integrated over a single tetrahedral element. They were run on a personal computer with a processor i7-3770 8x3.40 GHz with 16 GB of RAM memory.

Examples 2 and 3 deal with practical acoustic problems solved with the PUM enriched with plane waves. They have been computed in a machine Dell Power Edge R630 Xeon E5-2667 v3 (2x8x3.2 GHz/20MB cache, 2133Mhz FSB) with 62 GB of available memory.

In the legends of the figures, acronym SA denotes the solutions obtained with the Semi-Analytical integration rule, and similarly GL denotes the solutions obtained with a Gauss-Legendre quadrature. All the developments are coded in MATLAB R2013b.

\subsection{Integration of the product of polynomials and a complex exponentials over a tetrahedron}

The objective of this example is to test the performance of the semi-analytical rule by comparing its accuracy and CPU time consumption with those of the standard Gauss-Legendre quadrature. To this end, the product of smooth functions and a complex exponential is integrated over a single tetrahedron, see Equation (1). The tetrahedron is defined by nodes $\boldsymbol{x}_{1}=[1,1,1], \boldsymbol{x}_{2}=[2,0,0]$, $\boldsymbol{x}_{3}=[2,2,2]$ and $\boldsymbol{x}_{4}=[1,0,3]$. Vector $\boldsymbol{v}$ is written as $\boldsymbol{v}=v \times[1,1,1]$, with $v=\{2,4,10,20,40\}$, corresponding to $1.09,2.19,5.47,10.94$ and 21.88 wavelengths per element, respectively. We consider three non-oscillatory functions: $f_{1}(\boldsymbol{x})=1, f_{2}(\boldsymbol{x})=r^{2}(\boldsymbol{x})$ and $f_{3}(\boldsymbol{x})=r^{4}(\boldsymbol{x})$, where $r(\boldsymbol{x})$ is the distance from $\boldsymbol{x}$ to the reference point $\boldsymbol{x}_{0}=[0,0,0]$. We have computed those integrals 
using the semi-analytical rule with $n_{S A}=1,2, \ldots, 10$ integration points per dimension, and the Gauss-Legendre quadrature with $n_{G L}=3,5,7,10,15,20,30,40,50$ and 100 integration points per dimension.

We measure the accuracy of the integration rules by computing the relative error

$$
r_{I}=\frac{\left|I-I_{r e f}\right|}{\left|I_{r e f}\right|}
$$

where $I$ is the value computed using the corresponding method and $I_{r e f}$ is a reference value. Since Gauss-Legendre quadratures converge to the exact value of the integral when the number of integration points is increased [21], we set $I_{\text {ref }}$ equals to the value of the integral computed using $n_{G L}=300$ Gauss points per direction.

We have computed the relative error (32) of the integrals when the integrand is $f_{1}(\boldsymbol{x})=1$ using the semi-analytical rule for all the detailed values of the wavelengths per element and number of integration points per dimension, $n_{G L}$. For this integrand, the error is exclusively produced by the integration of the weights, and the interpolation error of the integrand is null (apart from roundoff errors) regardless the number of integration points. The rule produces excellent results and the relative error remains very low $\left(r_{I}<10^{-12}\right)$ for all cases. Nevertheless, the error is still lower for small values of $v$, for instance $r_{I}<10^{-14}$ for $v=2$ and $v=4$.

Figure 2 plots the relative error (32) of both integration methods against the integration CPU time for $f_{2}(\boldsymbol{x})=r^{2}(\boldsymbol{x})$ and $f_{3}(\boldsymbol{x})=r^{4}(\boldsymbol{x})$. We observe that, as we increase the number of Gauss points, the integrals calculated with the Gauss-Legendre quadrature tend to the reference value. The behavior of this quadrature is the same for both functions, requiring an increasing number of integration points to reach an acceptable accuracy when $v$ grows, especially if the integrand oscillates more than 10 times in an element $(v>20)$. The relative error of the semi-analytical rule drastically decreases when we use $n_{S A}=p+1$ integration points per dimension, $p$ being the degree of $f(\boldsymbol{x})$. That is, $n_{S A}=3$ and $n_{S A}=5$ integration points for $f(\boldsymbol{x})=r^{2}(\boldsymbol{x})$ and $f(\boldsymbol{x})=r^{4}(\boldsymbol{x})$, respectively. Note that, according to Equation (3), this number coincides with the minimum number of Lagrange points required to exactly approximate the integrand except for round-off errors. It is important to point out that for both functions, the semi-analytical rule outperforms the GaussLegendre quadratures when the integrands have more than 10 wavelengths per element $(v>20)$.

\section{Remark 4}

The main cost of the semi-analytical rule comes from the computation of the integration weights. In this sense, the scenario in the first example is unfavorable for the semi-analytical rule since only a single integral is computed with the same set of weights. This is not the case of several practical applications such as the PUM enriched with plane waves. In this case, the products of several polynomial functions and the same complex exponential function have to be integrated over the same reference element when computing the elemental contributions (31). Therefore, the weights can be computed once for each element, drastically reducing the computational cost of the semi-analytical rule. This reduction is obtained for any value of the number of oscillations in the integration interval. In the context of highly-oscillatory integrands, this approach is competitive compared with the Gauss-Legendre quadrature because it requires a much lower number of integration points. 


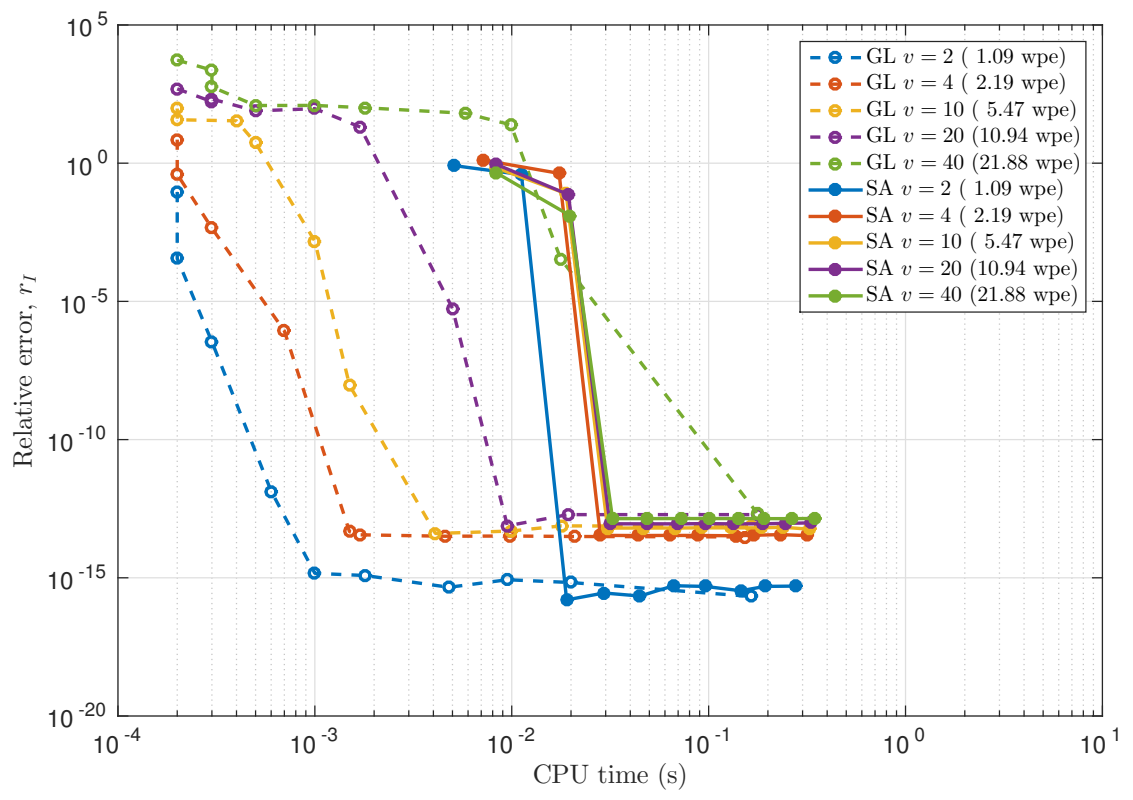

(a)

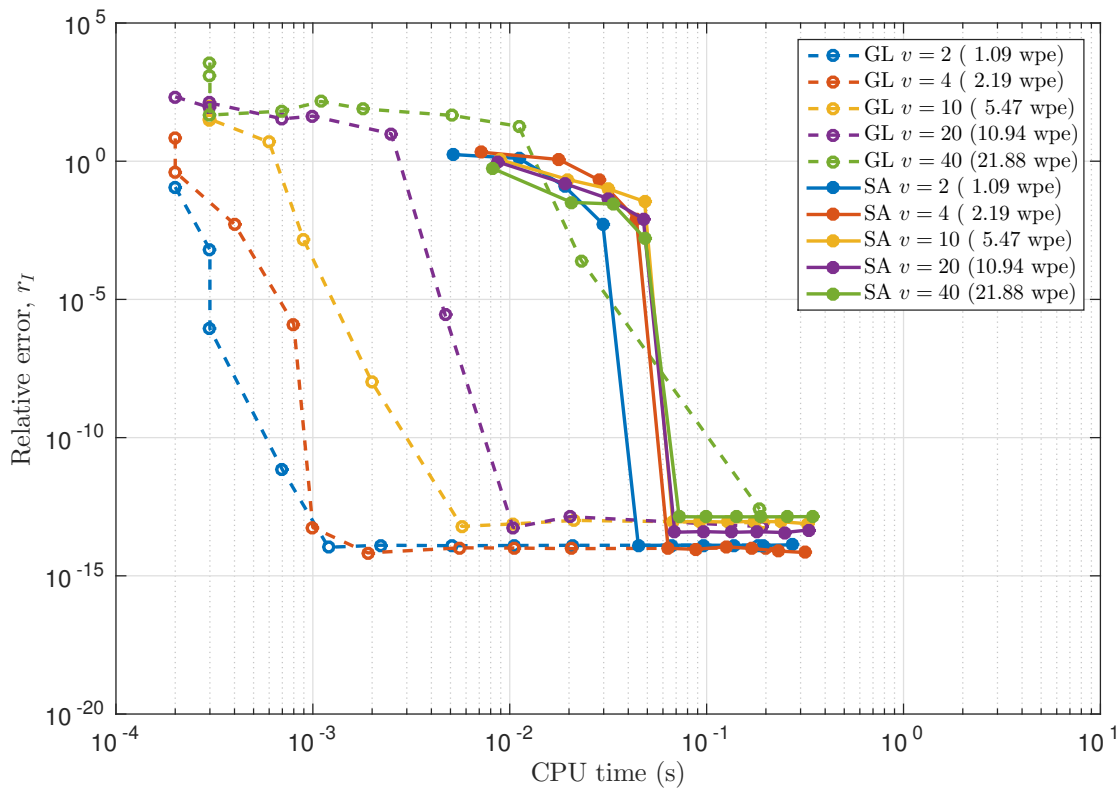

(b)

Figure 2. Relative error against integration CPU time for two non-oscillatory functions in Example 1: (a) $f_{2}(\boldsymbol{x})=r^{2}(\boldsymbol{x})$; (b) $f_{3}(\boldsymbol{x})=r^{4}(\boldsymbol{x})$. Number of integration points per dimension and element: $n_{S A}=$ $1,2, \ldots, 10 ; n_{G L}=3,5,7,10,15,20,30,40,50$ and 100 .

Figure 3 illustrates the behavior indicated in the previous remark, by plotting the evolution of the CPU time against the number of times the same integral is computed over a given element for the same level of accuracy. The time consumed by the Gauss-Legendre quadrature linearly increases with a rate of $1.6 \mathrm{~s}$ for every 1000 integrals. On the contrary, the CPU time for the semi-analytical 


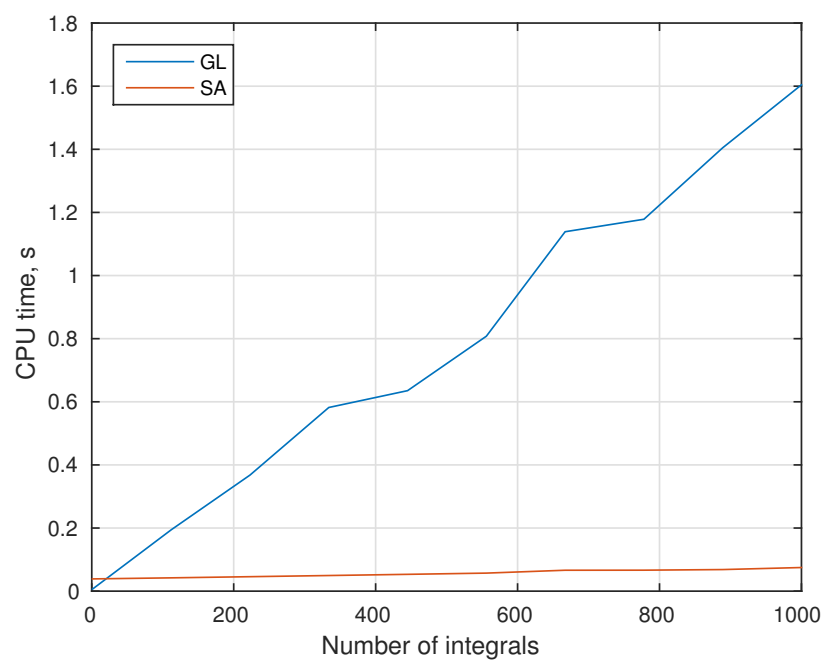

Figure 3. CPU time against number of integrals computed over the same element, with $v=30, n_{G L}=20$ and $n_{S A}=3$.

rule presents a moderate initial jump corresponding to the computation of the weights, and then it grows with a small rate, that is practically flat with respect to the Gauss-Legendre slope. The overhead cost for each integral computation is associated with 1 . the number of integration points and 2. the cost of functional evaluation at the integration points (recall that for Gauss-Legendre it involves computing costly complex exponential functions). Likewise, if the computational cost of evaluating the non-oscillatory function $f(\boldsymbol{x})$ is high, the Gauss-Legendre quadrature is further penalized since the integrand has to be evaluated a larger number of times.

\subsection{Simulation of a single traveling wave using PUM}

In this example we assess the accuracy and the performance of the semi-analytical rule in a practical application by comparing it with the standard Gauss-Legendre quadrature, when the propagation of a single time-harmonic traveling wave through a cubic domain is computed using the PUM enriched with plane waves.

First, we use the Frobenius norm to compare the global matrices obtained from the volumetric contributions of the weak form (27). Second, we solve the global linear systems, including the boundary conditions, and we compute the relative error in $L^{2}$-norm of the PUM solution with respect to the analytical solution. Specifically, we consider a single traveling plane wave with the following form:

$$
u(\boldsymbol{x})=A_{p w} \exp \left(i k \boldsymbol{e}_{p w} \cdot \boldsymbol{x}\right),
$$

where $A_{p w}=1 \mathrm{~Pa}$ is the amplitude, $k=0.52 \mathrm{~m}^{-1}$ is the wavenumber and $\boldsymbol{e}_{p w}=[0,0,1]$ is the direction vector of the wave (towards positive $z$-axis). The plane waves directions are obtained thanks to the algorithm developed by Leopardi [22]. This algorithm has been recently used in [19]. In this example, the direction of propagation of the solution matches one of the directions of the set of plane waves basis and, thus, the traveling wave (33) belongs to the solution space (28). This 


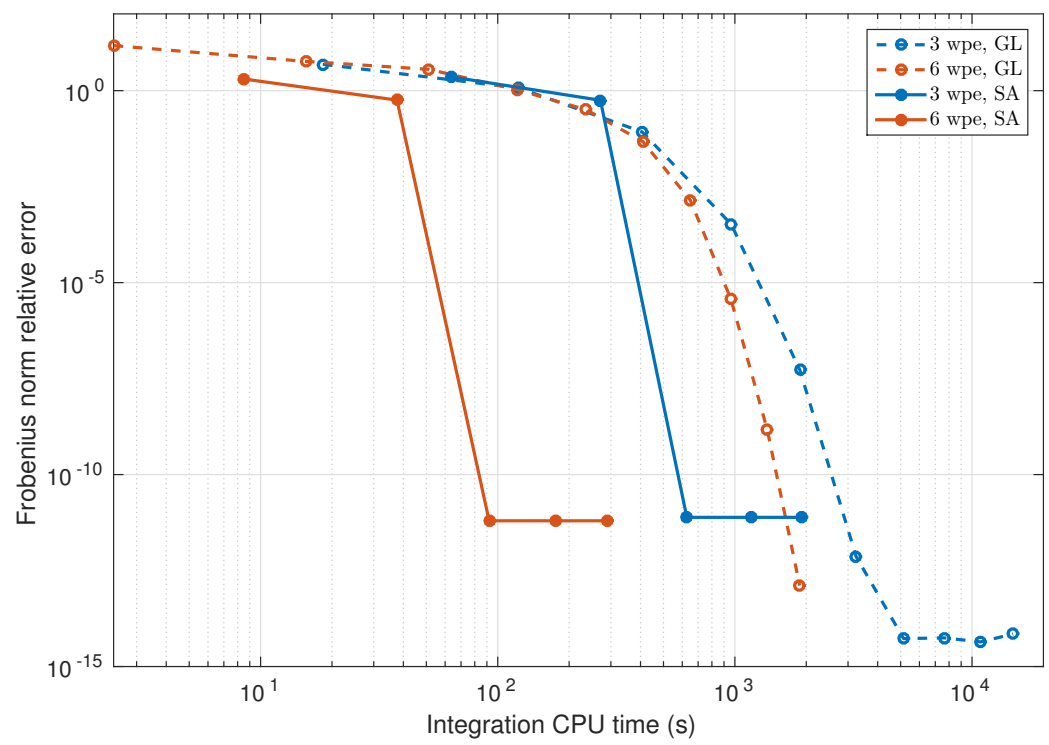

Figure 4. Relative error of the volume contribution to the system matrix in terms of the Frobenius norm against the integration CPU time in Example 2. Number of integration points per dimension and element: $n_{S A}=1, \ldots, 5 ; n_{G L}=5,10,15, \ldots, 50$.

cancels the error due to the quality of the approximation space, while focusing on the error produced during the integration step.

The size of the domain is $72 \times 72 \times 72 \mathrm{~m}$, and it is discretized using two different coarse meshes composed of 6 and 48 tetrahedra ( 6 and 3 wavelengths per element, respectively), pasting $q=150$ plane waves at each node of the meshes. We solve Equation (25) for both geometry discretizations, prescribing the appropriate boundary condition (26). Equation (33) is introduced into the Robin conditions (26) with $\tau=i$, and the resulting independent term $g$ is integrated over the whole boundary. We use the procedure proposed by Bettess et al. [17], summarized in Appendix C, to compute the 2D integrals. The volume contributions to the global system matrix (31) are computed using the semi-analytical rule with $n_{S A}=1, \ldots, 5$ integration points per dimension and element, and the Gauss-Legendre quadratures with $n_{G L}=5,10,15, \ldots, 50$ integration points per dimension and element.

First, the system matrices obtained with both methods are compared by computing the relative error

$$
r_{m}=\frac{\left\|\mathbf{K}_{I}-\mathbf{K}_{r e f}\right\|_{F}}{\left\|\mathbf{K}_{r e f}\right\|_{F}},
$$

where $\mathbf{K}_{I}$ is the system matrix computed using a numerical integration rule, $\mathbf{K}_{r e f}$ is the system matrix computed using a reference quadrature, and $\|\cdot\|_{F}$ denotes the Frobenius norm defined as

$$
\left\|\mathbf{A}_{(m \times n)}\right\|_{F}=\sqrt{\sum_{i=1}^{m} \sum_{j=1}^{n}\left|a_{i j}\right|^{2}},
$$

$|\cdot|$ being the complex modulus. We set as a reference value the matrix obtained using the GaussLegendre quadrature with $n_{G L}=80$ integration points per dimension and element. 


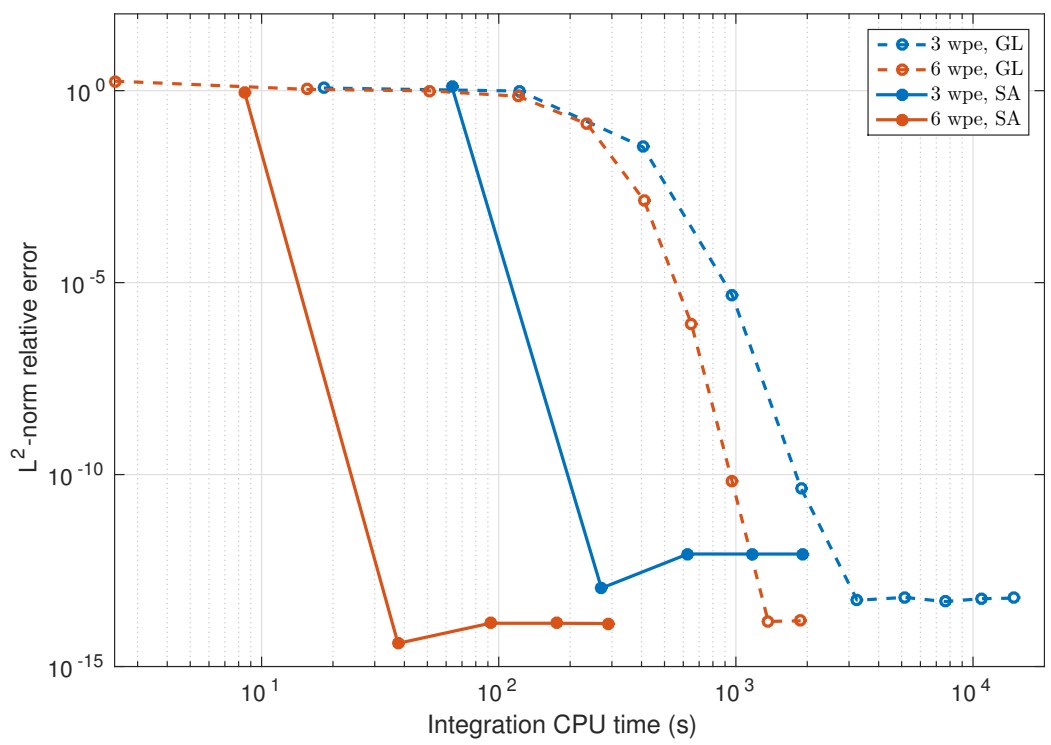

Figure 5. Relative error in $L^{2}$-norm against integration CPU time in Example 2. Number of integration points per dimension and element: $n_{S A}=1, \ldots, 5 ; n_{G L}=5,10,15, \ldots, 50$.

Figure 4 shows the relative error in terms of the Frobenius norm (34) versus the required CPU time for the integration step. We observe that the semi-analytical rule with using 3 or 4 integration points per dimension achieves an excellent accuracy $\left(r_{m}<10^{-11}\right)$ using less than one order of magnitude of the CPU time required by the Gauss-Legendre quadrature. As expected, we realize that using meshes with 6 waves per element (coarse meshes) is cheaper than using meshes with 3 waves per element (fine meshes). Note that this is also true for Gauss-Legendre quadratures. However, they require a larger number of integration points to achieve a similar accuracy. After solving the systems, we measure the accuracy of the numerical solutions by computing the relative error in $L^{2}$-norm with respect to the analytical solution (33) as

$$
r_{L^{2}}=\frac{\left\|u_{h, q}(\boldsymbol{x})-u_{a}(\boldsymbol{x})\right\|_{L^{2}}}{\left\|u_{a}(\boldsymbol{x})\right\|_{L^{2}}}
$$

where $u_{h, q}(\boldsymbol{x})$ is the numerical solution, $u_{a}(\boldsymbol{x})$ is the analytical solution (33), and

$$
\|\cdot\|_{L^{2}}=\sqrt{\int_{\Omega}|\cdot|^{2} \mathrm{~d} \Omega} .
$$

Figure 5 plots the relative error (35) of the numerical solutions versus the CPU time required by the integration step. We realize that the semi-analytical rule requires less than two orders of magnitude in CPU time to obtain a solution with the same accuracy than the obtained with the Gauss-Legendre quadrature. In addition, we also observe that using the semi-analytical procedure, the minimum error is achieved using 2 integration points per dimension and element. 
Table II. CPU time and relative error in $L^{2}$-norm in Example 3, for each of the three domains and both integration methods.

\begin{tabular}{cc|crc}
\hline Domain size $(\mathrm{m})$ & \# of elements (with PML) & Integration rule & \multicolumn{1}{c}{$t_{\text {int }}(\mathrm{s})$} & \multicolumn{1}{c}{$r_{L^{2}}$} \\
\hline \multirow{2}{*}{$36 \times 36 \times 18$} & \multirow{2}{*}{32} & GL & 4705.1 & $1.87 \mathrm{E}-002$ \\
& & SA & 579.9 & $1.88 \mathrm{E}-002$ \\
\hline \multirow{2}{*}{$72 \times 72 \times 18$} & \multirow{2}{*}{72} & GL & 19168.2 & $1.98 \mathrm{E}-002$ \\
& \multirow{2}{*}{192} & SA & 2367.0 & $1.94 \mathrm{E}-002$ \\
\hline \multirow{2}{*}{$108 \times 108 \times 36$} & & GL & 87185.0 & $6.01 \mathrm{E}-002$ \\
& & SA & 10752.6 & $6.01 \mathrm{E}-002$ \\
\hline
\end{tabular}

\subsection{Free-space propagation of a single noise source using PUM}

This example compares the proposed semi-analytical rule and the standard Gauss-Legendre quadrature in terms of accuracy and required CPU time, when the free propagation of underwater noise is computed using the PUM enriched with plane waves. We consider three cubic domains, see Table II, each of them is discretized using a tetrahedral mesh with 3 wavelengths per element, with $q$ $=330$ plane waves per node. The sound speed of the seawater is $1500 \mathrm{~ms}^{-1}$. The example considers time-harmonic waves with a frequency of $250 \mathrm{~Hz}\left(k=1.05 \mathrm{~m}^{-1}\right)$ generated by a single noise source located over the sea surface at $\boldsymbol{x}_{s}=[0,0, \lambda / 3]$, where $\lambda$ is the wavelength (note that the noise source is placed slightly outside of the domain to avoid the singularity, see [19, Section 2.5]). The intensity of the source is selected such that it produces a pressure modulus of $p_{0}=10 \mathrm{~Pa}$ at a reference distance from the source of $r_{0}=1 \mathrm{~m}$. The combination of the values for the frequency, the sound speed and the number of wavelengths per element produces an element size of $h=18 \mathrm{~m}$. The sea surface is treated as fully reflective, $\tau=0$, and the input noise is introduced through the independent term $g$ of the Robin condition, see Equation (26). Fully absorbing boundary conditions are prescribed at the lateral boundaries and sea bottom, using the PML technique [23]. The PMLs include a linear distribution for the attenuation parameter, characterized by a maximum value $\sigma_{\mathrm{PML}, \max }=30 \mathrm{~s}^{-1}$.

The analytical solution of the pressure field, $p_{a}(\boldsymbol{x})$, for this problem (homogeneous medium and uniform wavenumber) can be approximated by (see [24, Chapt. 5]):

$$
p_{a}(\boldsymbol{x})=p_{0} \frac{r_{0}}{r(\boldsymbol{x})} \exp \left(i k\left(r(\boldsymbol{x})-r_{0}\right)\right)
$$

where $r(\cdot)$ is the distance to the point source $\boldsymbol{x}_{s}$. We have performed the integration step of this problem by means of the semi-analytic rule with $n_{S A}=p+1=4$ integration points per element and dimension, since the non-oscillatory part of the integrands has a degree of $p=3$ (taking into account the variation in the weak form produced by the PMLs), and the Gauss-Legendre quadrature with 25 integration points per element and dimension. Figure 6 shows the real part of the pressure field for the second domain obtained with the semi-analytical rule.

The accuracy of the numerical solutions is measured by computing the relative error in $L^{2}$-norm, according to (35). Table II details the accuracy and the required CPU time using both integration methods. We notice that for each domain, and for the same level of accuracy, the CPU time required by the semi-analytical rule is almost one order of magnitude smaller than the time required by the Gauss-Legendre quadrature. 

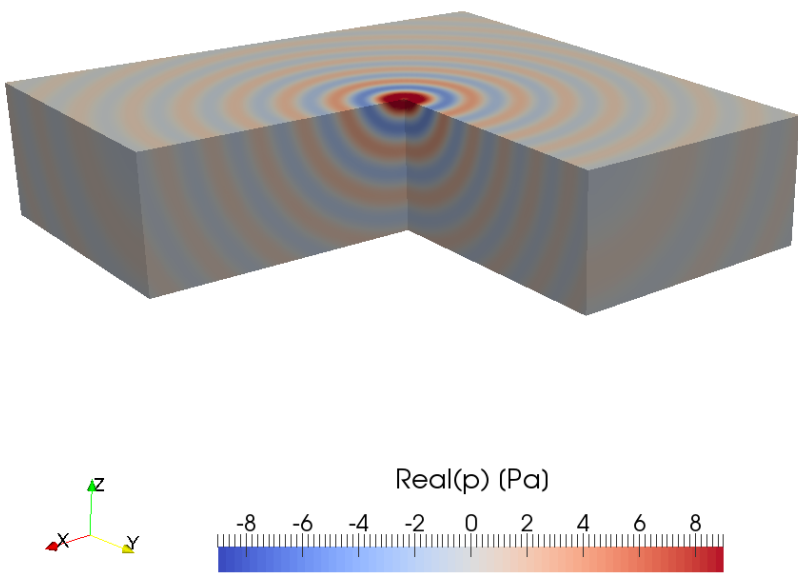

Figure 6. Real part of the pressure field obtained using the semi-analytical integration rule for the second domain in Example 3.

\section{CONCLUDING REMARKS}

In this work we detail a novel semi-analytical rule to compute the integral of highly oscillatory functions over a tetrahedron. The integrand is expressed as the product of a non-oscillatory part and a complex exponential function that models the oscillatory part. The rule is designed to be exact, except round-off errors, for integrals with a polynomial non-oscillatory part. This is of interest for a wide range of applications such us the numerical solution of the Helmholtz equation by the PUM enriched with plane waves. In these cases, $p+1$ integrations points per dimension should be considered, $p$ being the degree of the polynomial.

The key point of the proposed rule is to approximate the non-oscillatory part using Lagrange interpolation, that is an exact representation (up to round-off errors) for polynomials. Then, the Lagrange polynomials are linearly decomposed in terms of the appropriate set of monomials. In order to integrate the products of these monomials with a complex exponential over a tetrahedral element, our procedure identifies 16 possible cases that lead to a collection of one-dimensional highly oscillatory integrals. For these kind of integrals we propose a scheme that selects an appropriate 1D analytical integration procedure that provides the required accuracy with a reduced computational cost.

The examples clearly show that the proposed rule efficiently integrates, both in terms of accuracy and CPU time, the product of a polynomial and a complex exponential function over tetrahedra. Specifically, it provides relevant CPU time savings for the same level of accuracy, compared to the standard Gauss-Legendre rules, in either of these situations: 1. when the integrands have more than 10 oscillations in the integration interval; 2 . when the semi-analytical rule is applied to compute multiple integrals with the same oscillatory part (same arguments for the complex exponential). In this case, the integration weights can be pre-computed producing substantial CPU time reductions. 
It is important to highlight that this is the case of the integrals arising in the plane waves enriched PUM.

It is worth to notice that if the evaluation of the integrand is computationally expensive, then the semi-analytical rule is even more competitive since it involves a smaller number of integration points than the Gauss-Legendre quadratures.

Several aspects of the proposed model should be investigated in the near future. We have used a set of evenly spaced points in our implementation of the semi-analytical rule. However, other distribution of points can be analyzed that provide better interpolation properties. In addition, we also plan to analyze the relative performance of this rule against the promising integration method presented by Gabard in [20]. Finally, further research is needed regarding the extension of the present rule to hexahedra or other three-dimensional elements.

\section{A. EXPANSION OF THE LAGRANGE POLYNOMIALS INTO SETS OF MONOMIALS}

This appendix details a procedure to compute the coefficients of the Lagrange polynomials decomposition into monomials. Our goal is to find the set of coefficients $\lambda_{d, a}$ such that

$$
L_{d}(\boldsymbol{\xi})=\sum_{|\boldsymbol{a}| \leq p} \lambda_{d, \boldsymbol{a}} \xi^{a} \eta^{b} \zeta^{c}, \quad \text { for } d=1, \ldots, n_{p},
$$

where $\boldsymbol{a}=(a, b, c), a, b, c$ being non-negative integers and $|\boldsymbol{a}|:=a+b+c \leq p$. Let

$$
\boldsymbol{m}(\boldsymbol{\xi})=\left(\begin{array}{c}
1 \\
\xi \\
\eta \\
\vdots \\
\zeta^{p}
\end{array}\right) \text { that corresponds to }\left\{\begin{array}{l}
\boldsymbol{a}=(0,0,0) \\
\boldsymbol{a}=(1,0,0) \\
\boldsymbol{a}=(0,1,0) \\
\vdots \\
\boldsymbol{a}=(0,0, p)
\end{array}\right.
$$

be the column vector containing the values of the $n_{p}$ monomials at point $\boldsymbol{\xi}$, and let

$$
l(\boldsymbol{\xi})=\left(\begin{array}{c}
L_{1}(\boldsymbol{\xi}) \\
L_{2}(\boldsymbol{\xi}) \\
\vdots \\
L_{n_{p}}(\boldsymbol{\xi})
\end{array}\right)
$$

be the column vector containing the values of the $n_{p}$ Lagrange polynomials at point $\boldsymbol{\xi}$. Therefore, the $n_{p}$ equations in (37) can be written using a matrix notation as

$$
l(\xi)=\Lambda m(\xi),
$$

where $\boldsymbol{\Lambda}$ is a square matrix collecting the $n_{p} \times n_{p}$ unknown coefficients $\Lambda_{(p, m(\boldsymbol{a}))}=\lambda_{p, \boldsymbol{a}}, m(\boldsymbol{a}) \in$ $\left\{1, \ldots, n_{p}\right\}$ being a scalar that assigns a given order to each monomial with multi-index $\boldsymbol{a}$ satisfying $|\boldsymbol{a}| \leq p$. 
Enforcing Equation (38) at the $n_{p}$ interpolation points we have

$$
\mathbf{L}=\Lambda \mathrm{M}
$$

where

$$
\mathbf{L}=\left(\begin{array}{ccc}
\vdots & & \vdots \\
l\left(\boldsymbol{\xi}_{1}\right) & \ldots & \boldsymbol{l}\left(\boldsymbol{\xi}_{n_{p}}\right) \\
\vdots & & \vdots
\end{array}\right), \quad \text { and } \quad \mathrm{M}=\left(\begin{array}{ccc}
\vdots & & \vdots \\
\boldsymbol{m}\left(\boldsymbol{\xi}_{1}\right) & \ldots & \boldsymbol{m}\left(\boldsymbol{\xi}_{n_{p}}\right) \\
\vdots & & \vdots
\end{array}\right)
$$

Taking into account that

$$
L_{n}\left(\boldsymbol{\xi}_{d}\right)=\left\{\begin{array}{ll}
0 & \text { if } n \neq d \\
1 & \text { if } n=d
\end{array} \quad \text { for } n, d=1, \ldots, n_{p}\right.
$$

we obtain

$$
\mathrm{Id}_{n_{p}}=\Lambda \mathbf{M}
$$

where $\operatorname{Id}_{n_{p}}$ is the identity matrix of order $n_{p}$. Hence, matrix $\Lambda$ can be obtained as

$$
\Lambda=\mathbf{M}^{-1}
$$

Note that matrix $\Lambda$ only depends on the set of interpolation points that are defined on the reference element. Thus, it is evaluated once for a given set of integration points.

Finally, it is important to point out that Equation (38) allows writing the integration weights from Equation (9) in a compact vectorial notation:

$$
\boldsymbol{w}=\boldsymbol{\Lambda} \int_{\Omega^{\mathrm{ref}}} \boldsymbol{m}(\boldsymbol{\xi}) \exp (i \boldsymbol{v} \cdot \boldsymbol{x}(\boldsymbol{\xi})) \mathrm{d} \boldsymbol{\xi}=\boldsymbol{\Lambda} \exp (i D) \int_{\Omega^{\mathrm{ref}}} \boldsymbol{m}(\boldsymbol{\xi}) \exp (i A \xi) \exp (i B \eta) \exp (i C \zeta) \mathrm{d} \boldsymbol{\xi} .
$$

\section{B. INTEGRATION OF DEFINITE 1D HIGHLY OSCILLATORY FUNCTIONS}

The computation of highly oscillatory integrals over a tetrahedron using the proposed semianalytical method involves the computation of a considerable number of one-dimensional highly oscillatory integrals with the following form, see Section 4:

$$
I_{(0,1)}=\int_{0}^{1} s^{\nu} \exp (i \mu s) \mathrm{d} s
$$

where $\nu$ is a non-negative integer and and $\mu$ a complex coefficient.

There are several methods to compute these integrals, either analytical or numerical. The accuracy and computational efficiency of some of them depend on the combination of both parameters $\mu$ and $\nu$. In this appendix we present a heuristic procedure to select a method for each combination of these parameters. Our objective is to reduce the CPU cost while keeping the relative error of these 1D integrals below $10^{-12}$. This will allow us computing the integral over the tetrahedron with an acceptable accuracy. 
Bakhvalov and Vasileva [25] proposed an accurate and robust method to compute integral (39). It gives an analytical expression when the non-oscillatory part of the integral is a polynomial functions, as in Equation (39), provided that at least $\nu+1$ points are used. However, in 3D real simulations involving medium or high frequencies the computational of the method becomes too expensive due to the high number of evaluations of Bessel functions.

To overcome this shortcoming, we propose to use equations (12) and (13) for combinations of $\mu$ and $\nu$ that allows achieving the desired accuracy. Note that the most expensive function involved in these expressions is the factorial function of natural numbers, that in fact, are precomputed.

From our experience, for values of $\mu$ whose modulus are not close to $0,|\mu| \nrightarrow 0$, the most efficient method in terms of CPU time is obtained by applying Equation (12):

$$
\int_{0}^{1} s^{\nu} \exp (i \mu s) \mathrm{d} s=\nu ! \sum_{r=0}^{\nu} \frac{i^{r-1}\left(\exp (i \mu)-\lim _{s \rightarrow 0} s^{\nu-r}\right)}{(\nu-r) ! \mu^{r+1}} .
$$

Unfortunately, expression (40) loses accuracy when the modulus of $\mu$ is small, $|\mu| \rightarrow 0$, as stated by Bettess et al. $[17,18]$. In addition, we highlight that when using PUM in 3D applications the value of $\nu$ is much more higher than in 2D applications. Therefore, the modulus of each one of the addends in Equation (40) may differ by several orders of magnitude. This lead to inaccurate results since it implies the subtraction of similar terms whose modulus are much higher than the result of this operation. Therefore, the criterion for using Equation (40) depends on the combination of both values $\mu$ and $\nu$. For this reason, we use Equation (40) when the following relations are satisfied:

$$
\begin{aligned}
|\mu| & >\text { tol }_{1} \\
\frac{\nu !}{|\mu|^{\nu}} & >\text { tol }_{2} \\
\frac{|\mu|^{\nu}}{\nu !} & >\text { tol }_{3} \\
\frac{[(1-a) \nu] !}{|\mu|^{(1-a) \nu}} & >\text { tol }_{4} \\
\frac{[(1-a) \nu] !|\mu|^{a \nu}}{\nu !} & >\text { tol }_{5}
\end{aligned}
$$

where $a \in[0,1]$. Based in our numerical experiments, we set $a=0.6$ and $\operatorname{tol}_{i}=10^{-1}$ for $i=1, \ldots, 5$ in order to obtain the desired accuracy while reducing the CPU time.

When any of the above conditions is not satisfied, and at the same time the modulus of $\mu$ is moderate, $|\mu|<\mu_{\text {th }}$, we apply Equation (13). Thus:

$$
\int_{0}^{1} s^{\nu} \exp (i \mu s) \mathrm{d} s=\sum_{r=0}^{r_{\max }} \frac{(i \mu)^{r}}{r !} \frac{\left(1-\lim _{s \rightarrow 0} s^{\nu+r+1}\right)}{\nu+r+1} .
$$

As stated in Section 3, the value of $r_{\max }$ is adjusted so that the truncation error in the series is of the order of the machine accuracy $\epsilon$. Our numerical experiments show that the number of addends in the truncated series, $r_{\max }$, depends on the value of $\mu_{\mathrm{th}}$. Therefore, in order to limit it, and to reduce the computational cost of the integration step, we set $\mu_{\mathrm{th}}=4$.

When the values of $\mu$ and $\nu$ do not satisfy the conditions to use Equation (40) nor Equation (41), our implementation resorts to the Bakhvalov and Vasileva (BV) method [25] due to its accuracy 
and robustness. This method is more expensive from the computational point of view than the previous analytical methods, equations (40) and (41), but it provides more accurate results. Next we summarize the BV method for completeness, and we also detail an alternative expression to preserve the accuracy while reducing the CPU cost when the modulus of $\mu$ is small.

The BV method is based on an extension of the Gauss-Legendre rule to the highly oscillatory case. It is designed to compute efficiently the following type of definite integral:

$$
I_{\mathrm{BV}}(f, \Omega)=\int_{-1}^{+1} f(x) \exp (i \Omega x) \mathrm{d} x
$$

where $f(x)$ is a non-oscillatory (smooth) function. This function is approximated by a set of $n_{L}+1$ Legendre polynomials:

$$
f(x) \approx \sum_{d=0}^{n_{L}} c_{d} P_{d}(x)
$$

where $P_{d}(x)$ is the Legendre polynomial of degree $d$ in $[-1,+1]$. The coefficient $c_{d}$ is obtained as

$$
c_{d}=\left(\frac{2 d+1}{2}\right) \sum_{m=0}^{\nu} w_{m} P_{d}\left(x_{m}\right) f\left(x_{m}\right)
$$

where $x_{m}$ and $w_{m}$ are the $m$-th point and weight of the Gauss-Legendre quadrature of $\left(n_{L}+1\right)$ points, respectively. Taking into account that

$$
\int_{-1}^{+1} P_{d}(x) \exp (i \Omega x) d x=i^{d}\left(\frac{2 \pi}{\Omega}\right) J_{d+1 / 2}(\Omega)
$$

where $J_{d}(\Omega)$ is the Bessel function of the first kind and order $d$, we have

$$
\int_{-1}^{+1} f(x) \exp (i \Omega x) \mathrm{d} x \approx \sum_{d=0}^{n_{L}} i^{d}(2 d+1)\left(\frac{\pi}{2 \Omega}\right)^{1 / 2} J_{d+1 / 2}(\Omega)\left(\sum_{m=0}^{n_{L}} w_{m} P_{d}\left(x_{m}\right) f\left(x_{m}\right)\right) .
$$

The main drawback of Equation (43) is that its accuracy is reduced when the value of $\Omega$ is small, $|\Omega| \rightarrow 0$. To mitigate this issue, we consider the Taylor expansion of the Bessel function for small values of $\Omega$. In this case we have

$$
\frac{J_{d+1 / 2}(\Omega)}{\Omega^{1 / 2}} \approx \frac{1}{\Gamma(d+3 / 2)} \frac{\Omega^{d}}{2^{d+1 / 2}}
$$

where $\Gamma(\cdot)$ is the Gamma function. Note that in Equation (44) the Gamma function depends on a natural number. Therefore, their values can be precomputed, reducing the computational cost of Equation (43) when $|\Omega| \rightarrow 0$. Based on our experience we consider the threshold value $\Omega_{\mathrm{th}}=10^{-7}$.

In order to apply the BV method to compute Equation (39) we consider the transformation $s(x)=(x+1) / 2$, giving

$$
I_{(0,1)}=\frac{1}{2} \exp (i \mu / 2) I_{\mathrm{BV}}\left(s^{\nu}(x), \mu / 2\right) .
$$

Finally, note that when $f(x)$ is a polynomial of degree $n_{L}$, the expansion in (42) is exact. Thus, the $\mathrm{BV}$ method with $n_{L}+1$ points is analytical and provides the exact value of the integral except (up to round-off errors). 
Table III. Possible combinations of the values of coefficients $B$ and $C$ in the semi-analytical rule for triangles, generating 5 special cases.

\begin{tabular}{c|ccc}
\hline Case & $B$ & $C$ & $C-B$ \\
\hline 1 & $\nrightarrow 0$ & $\nrightarrow 0$ & $\nrightarrow 0$ \\
2 & $\nrightarrow 0$ & $\nrightarrow 0$ & $\rightarrow 0$ \\
3 & $\nrightarrow 0$ & $\rightarrow 0$ & - \\
4 & $\rightarrow 0$ & $\nrightarrow 0$ & - \\
5 & $\rightarrow 0$ & $\rightarrow 0$ & - \\
\hline
\end{tabular}

\section{SEMI-ANALYTICAL RULE FOR TRIANGULAR ELEMENTS}

In Section 4.1, the analytical development of cases 1-11, $A \nrightarrow 0$, leads to Equation (16), which includes the following 2D integral

$$
I_{\mathrm{TRI}}=\int_{0}^{1} \int_{0}^{1-\zeta} \eta^{b} \zeta^{c} \exp (i B \eta) \exp (i C \zeta) \mathrm{d} \eta \mathrm{d} \zeta .
$$

Bettess et al. [17] developed a semi-analytical rule to compute this type of integrals. For the sake of consistency, we summarize it here detailing our implementation and preserving the notation used in our work. They identify 5 cases, see Table III, depending on the values of $B, C$ and $C-B$.

C.1. Cases from 1 to $3(B \nrightarrow 0)$

If $B \nrightarrow 0$, Equation (12) is introduced into Equation (45) leading to

$$
\begin{array}{r}
I_{\mathrm{TRI}}=b !\left(\exp (i B) \sum_{u=0}^{b}\left(\frac{i^{u-1}}{(b-u) ! B^{u+1}} \int_{0}^{1} \zeta^{c}(1-\zeta)^{b-u} \exp (i(C-B) \zeta) \mathrm{d} \zeta\right)\right. \\
\left.-\frac{i^{b-1}}{B^{b+1}} \int_{0}^{1} \zeta^{c} \exp (i C \zeta) \mathrm{d} \zeta\right)
\end{array}
$$

The binomial theorem, Equation (14), is used to develop the first integral. Then, we apply the procedure presented in Appendix B to the resulting expression, depending on the values of the resulting monomial exponents and $C-B$. Similarly, for the second integral we use the procedure detailed in Appendix B depending on the values of $c$ and $C$.

C.2. Cases 4 and $5(B \rightarrow 0)$

If $B \rightarrow 0$, Equation (13) is introduced into Equation (45), leading to

$$
I_{\mathrm{TRI}} \approx \sum_{u=0}^{u_{\max }} \frac{(i B)^{u}}{u !(b+u+1)} \int_{0}^{1} \zeta^{c}(1-\zeta)^{b+u+1} \exp (i C \zeta) \mathrm{d} \zeta .
$$

The binomial theorem, Equation (14), is used again to develop this integral. Then, the procedure presented in Appendix B is applied depending on the values of the monomial exponents and $C$. 
1. Babuška IM, Sauter SA. Is the pollution effect of the fem avoidable for the helmholtz equation considering high wave numbers? SIAM Journal on Numerical Analysis 1997; 34(6):2392-2423, doi:10.1137/S0036142994269186.

2. Ihlenburg F. Finite Element Analysis of Acoustic Scattering, Applied Mathematical Sciences, vol. 132. 1st edn., Springer-Verlag: New York, 1998.

3. Deraemaeker A, Babuška I, Bouillard P. Dispersion and pollution of the fem solution for the helmholtz equation in one, two and three dimensions. International Journal for Numerical Methods in Engineering 1999; 46(4):471-499, doi:10.1002/(SICI)1097-0207(19991010)46:4;471::AID-NME684¿3.0.CO;2-6.

4. Melenk JM. On generalized finite element methods. PhD Thesis, The University of Maryland 1995.

5. Melenk J, Babuška I. The partition of unity finite element method: Basic theory and applications. Computer Methods in Applied Mechanics and Engineering 1996; 139(14):289 - 314, doi:10.1016/S0045-7825(96)01087-0.

6. Farhat C, Harari I, Franca LP. The discontinuous enrichment method. Computer methods in applied mechanics and engineering 2001; 190(48):6455-6479.

7. Farhat C, Harari I, Hetmaniuk U. A discontinuous galerkin method with lagrange multipliers for the solution of helmholtz problems in the mid-frequency regime. Computer Methods in Applied Mechanics and Engineering 2003; 192(11):1389-1419.

8. Farhat C, Tezaur R, Weidemann-Goiran P. Higher-order extensions of a discontinuous galerkin method for midfrequency helmholtz problems. International journal for numerical methods in engineering 2004; 61(11):19381956.

9. Hiptmair R, Moiola A, Perugia I. Plane wave discontinuous galerkin methods for the $2 \mathrm{~d}$ helmholtz equation: analysis of the p-version. SIAM Journal on Numerical Analysis 2011; 49(1):264-284.

10. Herrera I, Sabina FJ. Connectivity as an alternative to boundary integral equations: Construction of bases. Proceedings of the National Academy of Sciences 1978; 75(5):2059-2063.

11. Laghrouche O, Bettes P. Short wave modelling using special finite elements. Journal of Computational Acoustics 2000; 8(01):189-210.

12. Cessenat O, Després B. Application of an ultra weak variational formulation of elliptic pdes to the two-dimensional helmholtz problem. SIAM journal on numerical analysis 1998; 35(1):255-299.

13. Evans G, Webster J. A comparison of some methods for the evaluation of highly oscillatory integrals. Journal of Computational and Applied Mathematics Nov 1999; 112(1-2):55-69, doi:10.1016/S0377-0427(99)00213-7.

14. Iserles A, Nørsett SP. Efficient quadrature of highly oscillatory integrals using derivatives. Proceedings of the Royal Society of London A: Mathematical, Physical and Engineering Sciences 2005; 461(2057):1383-1399, doi: 10.1098/rspa.2004.1401.

15. Huybrechs D, Vandewalle S. On the evaluation of highly oscillatory integrals by analytic continuation. SIAM Journal on Numerical Analysis 2006; 44(3):1026-1048, doi:10.1137/050636814.

16. Ortiz P, Sanchez E. An improved partition of unity finite element model for diffraction problems. International Journal for Numerical Methods in Engineering Feb 2001; 50(12):2727-2740, doi:10.1002/nme.161.

17. Bettess P, Shirron J, Laghrouche O, Peseux B, Sugimoto R, Trevelyan J. A numerical integration scheme for special finite elements for the helmholtz equation. International Journal for Numerical Methods in Engineering Nov 2003; 56(4):531-552, doi:10.1002/nme.575.

18. Sugimoto R, Bettess P, Trevelyan J. A numerical integration scheme for special quadrilateral finite elements for the helmholtz equation. Communications in Numerical Methods in Engineering Dec 2003; 19(3):233-245, doi: $10.1002 / \mathrm{cnm} .584$.

19. Hospital-Bravo R, Sarrate J, Díez P. Numerical modeling of undersea acoustics using a partition of unity method with plane waves enrichment. Computational Mechanics 2016; 57(5):717-732, doi:10.1007/s00466-015-1257-8.

20. Gabard G. Exact integration of polynomial-exponential products with application to wave-based numerical methods. Communications in Numerical Methods in Engineering Mar 2009; 25(3):237-246, doi:10.1002/cnm.1123.

21. Davis PJ, Rabinowitz P. Methods of numerical integration, chap. 2.10.2. Second edn., Computer Science and Applied Mathematics, Academic Press, Inc.: San Diego, California, USA, 1984; 151-155.

22. Leopardi P. A partition of the unity sphere into regions of equal area and small diameter. Electronic Transactions on Numerical Analysis 2006; 25:309-327.

23. Berenger JP. A perfectly matched layer for the absorption of electromagnetic waves. Journal of Computational Physics Oct 1994; 114(2):185-200, doi:10.1006/jcph.1994.1159.

24. Kinsler LE, Frey AR, Coppens AB, Sanders JV. Fundamentals of acoustics. Fourth edn., John Wiley \& Sons: New York, USA, 1999.

25. Bakhvalov NS, Vasilčeva L. Evaluation of the integrals of oscillating functions by interpolation at nodes of gaussian quadratures. USSR Computational Mathematics and Mathematical Physics 1968; 8(1):241-249. 